\title{
Suggestion of End Points of Complete Keloid Excision
}

\author{
Tae Hwan Park $\cdot$ Choong Hyun Chang
}

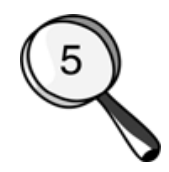

Received: 25 March 2012/ Accepted: 3 July 2012/Published online: 18 September 2012

(c) Springer Science+Business Media, LLC and International Society of Aesthetic Plastic Surgery 2012

Level of Evidence $V$ This journal requires that authors assign a level of evidence to each article. For a full description of these Evidence-Based Medicine ratings, please refer to the Table of Contents or the online Instructions to Authors www.springer.com/00266.

We read with great interest the article by Sunohara et al. [1] entitled "A Technique for Auricular Keloid Core Excision Using a Skin Biopsy Punch". The authors introduce their experiences with skin biopsy punch for the treatment of auricular keloids. They are to be commended for their efforts.

The authors believe that using a skin biopsy punch instead of a scalpel makes it easier and safer to perform keloid surgery. However, we do not use a scalpel when excising the keloid mass, except at the initial incision. Most of the time we perform the surgical excision with a sharp curved scissor [2-4].

We want to share two end points of complete keloid excision; we believe this information is useful when we use conventional scissors rather than a skin biopsy punch. First, when we excise a keloid lesion, the keloid tissue does not bleed profusely. On the other hand, complete excision with safety margins causes moderate marginal bleeding from the wound. For this reason, if we see sufficient marginal bleeding that requires step-by-step bipolar coagulation, it is a sign of complete excision. Second, during keloid tissue excision there typically is a crunching sound. This sound reflects the hardness of the keloid tissue. Hence, when we have performed a complete excision with safety margins,

T. H. Park $(\bowtie) \cdot$ C. H. Chang

Department of Plastic and Reconstructive Surgery, Kangbuk

Samsung Hospital, Sungkyunkwan University School of Medicine, 108 Pyung-Dong, Jongno-Gu, Seoul 110-746, Korea e-mail: hard-piano@hanmail.net we do not hear this sound any more. Thus, if we do not hear the typical sound and the excision is not difficult to perform, we have a complete excision.

We think the authors' novel technique is useful, especially for helical keloids. As the helix of the ear is a difficult area to perform a wide-wedge excision, step-by-step core excision using a skin biopsy punch can be helpful for many surgeons who are not familiar with auricular keloids. However, for lobular keloids, excision with a conventional scalpel and scissor can be done easily as surgical experience increases. In addition, to validate the authors' novel technique, a prospective study would be beneficial in establishing this method as an excellent option.

Conflict of interest The authors have no conflicts of interest to disclose.

\section{References}

1. Sunohara M, Ozawa T, Harada T et al (2012) A technique for auricular keloid core excision using a skin biopsy punch. Aesthetic Plast Surg 36(3):628-630

2. Park TH, Seo SW, Kim K et al (2011) Outcomes of surgical excision with pressure therapy using magnets and identification of risk factors for recurrent keloids. Plast Reconstr Surg 128:431-439

3. Park TH, Seo SW, Kim JK et al (2012) Earlobe keloids: classification according to gross morphology determines proper surgical approach. Dermatol Surg 38:406-412

4. Park TH, Seo SW, Kim JK et al (2012) Clinical characteristics of facial keloids treated with surgical excision followed by intra- and postoperative intralesional steroid injections. Aesthetic Plast Surg 36:169-173 